Pacific Journal of Mathematic

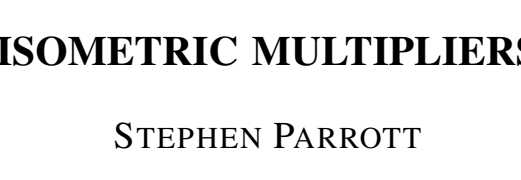




\title{
ISOMETRIC MULTIPLIERS
}

\author{
S. K. PARROTT
}

Let $G$ be a locally compact group with right Haar measure. A left multiplier on $L^{p}(G)$ is a bounded operator which commutes with all the operators induced by left translations. The main theorem of this paper states that every isometric left multiplier on $L^{p}(G)$ for $1 \leqq p<\infty, p \neq 2$, is a scalar multiple of an operator induced by a right translation.

Wendel proved this for $p=1$ and used it to show that if $L^{1}\left(G_{1}\right)$ and $L^{1}\left(G_{2}\right)$ are isomorphic as Banach algebras under convolution, then $G_{1}$ and $G_{2}$ are isomorphic as topological groups. In $\S 5$ we obtain some extensions of this result to $L^{p}$. An interesting byproduct is a theorem which states that an operator which is simultaneously a contraction on $L^{p}$ and unitary on $L^{2}$ (of a finite measure space) is actually an isometry on $L^{p}$.

Curiously, the proofs given below do not rely in any crucial way on the fact that the measure spaces $L^{p}(G)$ are defined with respect to Haar measure, and consequently the results are valid for a much larger class of measures. In $\S 4$ this fact is used to obtain examples of operators on $L^{p}$ which commute with no isometries (save scalar multiples of the identity).

An enlightening example is provided by taking $G$ to be the group of complex numbers of modulus one. It is not difficult to show that a multiplier on $L^{p}(G)$ sends a function $\sum_{n=-\infty}^{\infty} a_{n} z^{n}$ into $\sum_{n=-\infty}^{\infty} c_{n} a_{n} z^{n}$, where $\left\{c_{n}\right\}$ is a fixed sequence. If the multiplier is to be an isometry, each $c_{n}$ must have modulus one, and if $p=2$, this condition is also sufficient. For $p \neq 2, \infty$, the main theorem states that the multiplier is an isometry if and only if it is a scalar multiple of an operator induced by a rotation of the circle, which means there are constants $b, d$ of modulus one such that $c_{n}=d \cdot b^{n}$ for all $n$.

2. Preliminaries. Throughout, $G$ denotes a locally compact topological group with the group operation written multiplicatively. Elements of $G$ are indicated by $g, h, x, y, \cdots$, and Roman capitals $F, G, H, \cdots$, usually denote functions. The only $L^{p}$ spaces considered are those with $1 \leqq p<\infty$, and usually $p$ refers to a number in this range different from 2 . The $L^{p}$ spaces may be either real or complex, and all operators are assumed to be bounded. The characteristic function of the set $\Delta$ is called $\chi_{4}$.

The left and right translation operators $L_{g}$ and $R_{g}$ are defined by $\left(L_{g} F\right)(x)=F(g x)$ and $\left(R_{g} F\right)(x)=F(x g)$. We shall also denote the left 
translate $L_{g} F$ of $F$ by $F_{g}$.

The fact that the theorems to be presented are valid even if $L^{p}(G)$ is defined with respect to a measure other than Haar measure indicates that these results are more measure-theoretic than algebraic in nature. (In fact, if $\mu$ is not Haar measure, $L^{p}(G)$ is not even an algebra because convolution is not associative.) Essentially, they are consequences of the fact that there are relatively few isometries on $L^{p}$ of a measure space for $p \neq 2$.

This observation is probably more interesting than the particular generalizations thus obtained, so to avoid complications we shall restrict our attention to a smaller subclass of measures on $G$ than is strictly necessary. Specifically, we assume that the spaces $L^{p}(G)$ are defined with respect to a measure $\mu$ of the form $d u=\rho d \nu$, where $\nu$ is right Haar measure and $\rho$ is a positive function which is both bounded above and bounded away from zero. This hypothesis will not be stated separately in each theorem, and various properties of $\mu$ which follow from the corresponding properties of Haar measure will be used without comment.

We shall require an interesting theorem of Banach [1, Chapter 11], later refined and extended by Lamperti [5], which goes as follows. Let $(X, \mu)$ and $(Y, \nu)$ be measure spaces and $M$ an isometry from $L^{p}(X, \mu)$ into $L^{p}(Y, \nu), p \neq 2$. Then $M$ is of the form $S_{\varphi} U$, where, roughly, $S_{\varphi}$ is multiplication by a function and $U$ is induced by a "measurable transformation." More precisely, $\phi$ is a function on $Y$ whose restriction to any sigma-finite measurable set is measurable, and $S_{\varphi}$ is defined by $S_{\varphi}(F)=\varphi \cdot F$. The (possibly unbounded) operator $U$ is induced by a nonsingular isomorphism of the Boolean algebra of sigma-finite measurable sets in $(X, \mu)$ into the Boolean algebra of sigma-finite measurable sets in $(Y, \nu)$ (see [2], [5] for details). The pertinent facts about $U$ are that it sends characteristic functions into characteristic functions, preserves pointwise multiplication of $L^{\infty}$ functions $(U(F \cdot G)=(U F) \cdot(U G))$, and is an isometry of $L^{\infty}(X, \mu)$ into $L^{\infty}(Y, \nu)$. Usually, $U$ is induced by a point transformation $\tau$ from $Y$ onto $X:(U F)(y)=F(\tau y)$. (The statement of the theorem in [4] includes the hypothesis that $(X, \mu)$ and $(Y, \nu)$ be sigma-finite, but the extension to the situations to be encountered below is immediate.)

It is now easy to describe why every isometric multiplier is a scalar multiple of a right translation. For simplicity, assume that $\varphi(x)$ is never 0 and $U$ is induced by a point transformation $\tau$. Forget for the moment that measurable functions and transformations are only defined modulo sets of measure 0 . Then the relation $S_{\varphi_{g}} L_{g} U=$ $L_{g} S_{\varphi} U=S_{\varphi} U L_{g}$ suggests that $\varphi(g x) F(\tau(g x))=\varphi(x) F(g \cdot \tau x)$ for all $x, g$ in $G$ and $F$ in $L^{p}$. Consideration of this for characteristic functions 
$F$ suggests that $\varphi(g x)=\varphi(x)$ for all $x, g$ (hence $\varphi$ is constant), and $\tau$ commutes with left translations (hence $\tau$ is right translation by $\tau(e)$, where $e$ is the group identity). To make this rigorous, we shall transform "almost everywhere" considerations into pointwise ones via a standard result in the theory of commutative Banach algebras. This approach was suggested by Alessandro Figa-Talamanca.

\section{Isometric multipliers.}

THEOREM 1. Every left multiplier (not necessarily isometric) on $L^{p}(G, \mu), 1 \leqq p<\infty, p \neq 2$, of the form $S_{\varphi} U$ is a scalar multiple of a right translation $R_{g}$. In particular, every isometric left multiplier is a scalar multiple of a right translation.

Before proving this, we state a few simple lemmas. Lemmas 1 and 2 merely insure that measure-theoretic pathology cannot arise in the cases under consideration, and Lemma 3 is unnecessary if $S_{\varphi} U$ is assumed to map $L^{p}$ onto $L^{p}$. Thus the casual reader may profitably skip directly to the proof of Theorem 1 .

LEMma 1. Let $\phi$ be a function on $G$ such that for each sigmafinite set $E$,

(1) The restriction $\varphi \mid E$ of $\varnothing$ to $E$ is measurable.

(2) $\varphi\left|E=\varphi_{g}\right| E$ almost everywhere for each $g \in G$.

Then the restriction of $\varnothing$ to any sigma-finite set is constant almost everywhere, and the operator $S_{\varphi}$ is a scalar multiple of the identity.

Proof. For $M>0$, let $\varphi_{M}(X)=\varphi(x)$ or 0 according as $|\varphi(x)| \leqq M$ or $|\varphi(x)|>M$. Then $\varphi_{M}$ also satisfies (1) and (2). Let $\left\{V_{\alpha}\right\}$ be a basis of compact neighborhoods of the identity in $G$, and let $I_{\alpha}$ be the characteristic function of $V_{\alpha}$ divided by $\mu\left(V_{\alpha}\right)$. Then, as is well-known,

$$
\lim _{\alpha}\left(\varphi_{M} * I_{\alpha}\right)(x)=\lim _{\alpha} \int \varphi_{M}\left(x y^{-1}\right) I_{\alpha}(y) d \mu(y)
$$

exists for each $x$, and if $\Psi_{M}$ denotes the limit function, $\Psi_{M}$ agrees with $\varphi_{M}$ almost everywhere on each sigma-finite set. But $\Psi_{M}$ is clearly identically constant, since $\Psi_{M}(x)=\Psi_{M}(g x)$ for all $x, g \in G$, and hence $\varphi_{M}$ and $\varphi$ are constant almost everywhere on each sigma-finite set.

Lemma 2. Let $\varphi$ be a function in $L^{\infty}(G)$ such that given $\varepsilon>0$, there is a neighborhood $V$ of the identity such that for all $g \in V$, $\left\|\varphi-\varphi_{g}\right\|_{\infty}<\varepsilon$. Then $\varphi$ coincides almost everywhere with some left 
uniformly continuous function $\Psi$.

Proof. Again, set $\Psi(x)=\lim _{\alpha}\left(\varphi * I_{\alpha}\right)(x)$.

Lemma 3. Let $M$ be a nonzero multiplier on $L^{p}(G)$, and let $\Delta$ be a set of positive measure. Then there is an $F$ in $L^{p}$ such that the intersection of $\triangle$ and the support of $M F$ is nonzero. In particular, if $M=S_{\varphi} U$, the restriction of $\varphi$ to any sigma-finite set is nonzero almost everywhere.

Proof. If $F \in L^{p}$, then $L_{g} F \in L^{p}$, and $M\left(L_{g} F\right)=L_{g}(M F)$. The support of $L_{g}(M F)$ is $g^{-1}$ times the support of $M F$. If the support of $M F$ has positive measure, then there is a $g \in G$ such that $g^{-1}$ times the support of $M F$ intersects $\Delta$ in a set of positive measure $[4$, p. 260 , Th. E].

Proof of Theorem 1. We have $S_{\varphi}\left(U L_{g}\right)=L_{g} S_{\varphi} U=S_{\varphi_{g}}\left(L_{g} U\right)$. If $\Delta$ is a set with $0<\mu(\Delta)<\infty$, then $\chi_{\Delta} \in L^{p}$, and $\varphi \cdot\left(U L_{g} \chi_{\Delta}\right)=\varphi_{g} \cdot\left(L_{g} U \chi_{A}\right)$.

Because both $\left(U L_{g}\right) \chi_{\Delta}$ and $\left(L_{g} U\right) \chi_{\Delta}$ are characteristic functions and $\varphi$ is nonzero a.e. (Lemma 3 ), they are characteristic functions of the same set, say $\Delta^{\prime}$. Thus for each $g$ in $G, \varnothing=\phi_{g}$ almost everywhere on each set of the form $\Delta^{\prime}$ with $\chi_{\Delta^{\prime}}=\left(U L_{g}\right) \chi_{\Delta}=U\left(\chi_{g-1.4}\right)$, $0<\mu(\Delta)<\infty$. The class of sets $\Delta$ with $0 \leqq \mu(\Delta)<\infty$ is mapped onto itself by left translation, so $\varphi=\varphi_{g}$ almost everywhere on each set of the form $\Delta^{\prime}$ with $\chi_{\alpha^{\prime}}=U \chi_{A}, 0<\mu(\Delta)<\infty$. Given $g \in G$, if $\Lambda$ is a measurable set such that $\varphi(x) \neq \varphi(g x)$ for $x$ in $\Lambda$, then $\Lambda$ is disjoint from all sets of the form $\Delta^{\prime}$ above, and hence $A$ is disjoint from the support of every $U F$ and $S_{\varphi} U F$ with $F \in L^{p}$. Lemma 3 implies that $\Lambda$ has measure 0 , and Lemma 1 shows that $S_{\varphi}$ is a scalar multiple of the identity.

Let $F$ be a continuous function with compact support $\Delta$. Then $F$ is left uniformly continuous, and the relation $\left\|U F-(U F)_{g}\right\|_{\infty}=$ $\left\|U F-U\left(F_{g}\right)\right\|_{\infty}=\left\|F-F_{g}\right\|_{\infty}$ together with Lemma 2 show that $U F$ coincides almost everywhere with a unique left uniformly continuous function which we shall call $\hat{U} F$. Further, $\hat{U} F$ has compact support because $F \cdot F_{g}=0$ for all $g$ not in the compact set $\Delta \cdot \Delta^{-1}$ and thus $(\hat{U} F) \cdot(\hat{U} F)_{g}=\hat{U}\left(F \cdot F_{g}\right)=0$ for all $g \notin \Delta \cdot \Delta^{-1}$. (The support of $\hat{U} F$ is contained in $\Delta \cdot \Delta^{-1} \cdot x$, where $x$ is any point in the support of $\hat{U} F$.)

The Banach algebra $C_{0}(G)$ consisting of all continuous functions on $G$ vanishing at infinity (with the supremum norm) is generated by the set of continuous functions with compact support, and the preceding remarks show that $\hat{U}$ is an isometric isomorphism of $C_{0}(G)$ into itself which commutes with translations. It is known that each 
homomorphism of $C_{0}(G)$ into the complex numbers is of the form $\Psi_{g}$, where $\Psi_{g}(F)=F(g)[6$, p. 123]. Therefore if $e$ is the group identity, the homomorphism $\Psi_{e} \circ \hat{U}$ is $\Psi_{h}$ for some $h \in G$. For each $F \in C_{0}(G)$, $F(h)=\Psi_{h}(F)=\left(\Psi_{e} \circ \hat{U}\right)(F)=(\hat{U} F)(e)$. And, for any $g \in G$,

$$
(\hat{U} F)(g)=\left(L_{g} \hat{U} F\right)(e)=\left(\hat{U} L_{g} F\right)(e)=\left(L_{g} F\right)(h)=F(g h) .
$$

Therefore, $\hat{U}=R_{h}$ and also $U=R_{h}$ because $C_{0}(G)$ is dense in $L^{p}(G)$.

4. A class of operators which commute with no isometries. Theorem 1 states that every isometry on $L^{p}(G, \mu), p \neq 2$, which commutes with all left translations is a scalar multiple of a right translation. Of course, if $\mu$ is not right Haar measure, not all right translations, will be isometries. If $\mu$ is a measure such that no right translation $R_{g}$ with $g \neq e$ is an isometry, then no isometries except scalar multiples of the identity commute with all left translations. Thus if $\mu$ is of this type and $L_{g}$ is a left translation whose powers are dense in the weak operator topology in the set of all left translations, $L_{g}$ commutes with no nontrivial isometry.

It is easy to construct such situations. For instance, let $G$ be the group of complex numbers of modulus one with a measure $\mu$ defined by $d \mu=\varphi d \nu$, where $\nu$ is Lebesque measure and $\varphi(z)=1$ or 2 according as $z$ is on the upper or lower half circle. Clearly, no nontrivial translation is an isometry on $L^{p}(G, \mu)$. If $c$ is not a root of unity, the powers of the operator generated by the translation $z \rightarrow c \cdot z$ are easily shown to be dense in the group of translation operators, and hence this operator commutes with no nontrivial isometry on $L^{p}(G, \mu), p \neq 2$.

\section{Isomorphisms of convolution algebras.}

THEOREM 2. Let $G_{1}$ and $G_{2}$ be locally compact groups with respective measures $\mu_{1}, \mu_{2}$ as described in $\S 2$. Let $T$ be an isometry of $L^{p}\left(G_{1}, \mu_{1}\right)$ onto $L^{p}\left(G_{2}, \mu_{2}\right), 1 \leqq p<\infty, p \neq 2$, such that $T(F * G)=$ $T F * T G$ whenever $F * G \in L^{p}\left(G_{1}\right)$, and $T^{-1}(F * G)=\left(T^{-1} F\right) *\left(T^{-1} G\right)$ whenever $F * G \in L^{p}\left(G_{2}\right)$. Then there is a bicontinuous isomorphism $\tau$ of $G_{2}$ onto $G_{1}$. Further, if $\mu_{1}$ and $\mu_{2}$ are right Haar measures, there is a character $\lambda$ on $G_{2}$ and a positive constant $c$ such that $(T F)(g)=c \lambda(g) F(\tau g)$ for all $g \in G_{2}$.

This theorem was proved for $p=1$ and Haar measures $\mu_{1}, \mu_{2}$ by Wendel [7]. A later paper [9] gave a simpler proof and extended the theorem to the case in which $T$ is only assumed to be norm. decreasing. The solution of the isometric multiplier problem for $p \geqq 1$ 
(Theorem 1) enables us to easily adapt Wendel's later proof to establish Theorem 2. Only a sketch of the proof will be given here, and the reader may consult [9] for details.

Sketch of proof of Theorem 2. Let $\nu_{1}$ and $\nu_{2}$ be right Haar measures for $G_{1}$ and $G_{2}$ respectively, and suppose $d \mu_{1}=\rho_{1} d \nu_{1}, d \mu_{2}=$ $\rho_{2} d \nu_{2}$. Easy computations show that for any $F, G \in L^{p}\left(G_{1}, \mu_{1}\right)$, $L_{g}(F * G)=\left(L_{g} F\right) * G$ and $R_{g}(F * G)=F *\left(S R_{g} G\right)$, where $S(F)=\left(R_{g} \rho_{1} / \rho_{1}\right) \cdot F$. Further for any $g \in G_{1}$ and $F, G \in L^{p}\left(G_{2}, \mu_{2}\right)$,

$$
\left(T R_{g} T^{-1}\right)(F * G)=F *\left(T S R_{g} T^{-1} G\right) .
$$

Thus, it is apparent that $T R_{g} T^{-1}$ is a left multiplier on $L^{p}\left(G_{2}, \mu_{2}\right)$. If $T=S_{\varphi} U$ as described in $\S 1, T R_{g} T^{-1}=S_{\psi}\left(U R_{g} U^{-1}\right)$, where $\Psi=$ $\varphi \cdot\left(U R_{g} U^{-1}\left(\Phi^{-1}\right)\right)$. Now $U R_{g} U^{-1}$ is an operator induced by a Boolean set map, so by Theorem $1, T R_{g} T^{-1}$ is a scalar multiple of the operator induced by a right translation on $L^{p}\left(G_{2}\right)$. Define a map $\tau$ from $G_{2}$ onto $G_{1}$ and a function $\lambda$ on $G_{2}$ by $T R_{\tau g} T^{-1}=\lambda(g) R_{g}$. The proof that $\tau$ is a bicontinuous isomorphism from $G_{2}$ onto $G_{1}$ and the rest is now identical to that in [9].

Wendel established Theorem 2 under the weaker hypothesis that $\|T\| \leqq 1$ by first proving that any convolution-preserving contraction of $L^{1}\left(G_{1}\right)$ onto $L^{1}\left(G_{2}\right)$ is automatically an isometry. The author does not know if this is true in general for $L^{p}, p \neq 2$, but a more modest result can be obtained quite simply. First we make the following observation, which is perhaps of interest in its own right. The $L^{p}$ norm of a function $F$ is denoted by $\|F\|_{p}$.

Theorem 3. Let $(X, \mu)$ and $(Y, \nu)$ be measurable spaces with $\mu(X)=\mu(Y)<\infty$, and let $1 \leqq p<q \leqq \infty$. Suppose $T$ is an isometry of $L^{p}(X, \mu)$ into $L^{p}(Y, \nu)$ such that for each $F$ in $L^{q}(X, \mu),\|T F\|_{q} \leqq$ $\|F\|_{q}$. Then $T$ is an isometry of $L^{r}(X, \mu)$ into $L^{r}(Y, \nu)$ for all $r$, $1 \leqq r \leqq \infty$. In fact, $T$ is of the form $S_{\varphi} U$ described in $\S 2$, with $U$ induced by a measure-preserving transformation and $|\varphi|=1$.

Proof. We assume the measure spaces are normalized so that $\mu(X)=\nu(Y)=1$. A simple application of Holder's inequality shows that for all $F \in L^{p},\|F\|_{p} \leqq\|F\|_{q}$, and equality occurs if and only if $F$ has constant modulus one. For,

$$
\|F\|_{p}^{p}=\int|F|^{p} \leqq\left(\int\left(|F|^{p}\right)^{q / p}\right)^{p / q} \cdot \quad\left(\int 1^{q / q-p}\right)^{q-p / q}=\|F\|_{q}^{p} .
$$

If $F$ has modulus one, $\|F\|_{p}=\|F\|_{q}$, and by hypothesis

$$
\|T F\|_{q} \leqq\|F\|_{q}=\|F\|_{p}=\|T F\|_{p} \text {. }
$$


Hence $\|T F\|_{q}=\|T F\|_{p}$ and $T F$ has constant modulus one. If $\Delta$ is any set, and $|c|=1,\left|\chi_{\Delta}+c \chi_{X-\Delta}\right|=1$ a.e. and $\left|T \chi_{\Delta}+c T \chi_{X-\Delta}\right|=1$ a.e. This can happen for all $|c|=1$ only if $T \chi_{\Delta}$ and $T \chi_{X-4}$ have disjoint supports.

Let $e$ be the function constantly one, and let $U=S_{T_{(e)}^{-1}}^{-1} T$. The new operator $U$ satisfies the hypotheses because $|T(e)|=1$. Now $U \chi_{\Delta}+U \chi_{X-4}=U e=e$, and $U \chi_{\Delta}$ and $U \chi_{X-4}$ have disjoint supports, so $U \chi_{\Delta}$ is a characteristic function. Hence if $F=\sum c_{i} \chi_{E_{i}}$ is a simple function with $E_{i}$ pairwise disjoint, then for all $r \geqq 1$,

$$
\begin{aligned}
\|U F\|_{r}^{r} & =\int|U F|^{r} d \nu=\sum\left|c_{i}\right|^{r} \int\left|U \chi_{E_{i}}\right|^{r} d \nu \\
& =\sum\left|c_{i}\right|^{r} \int\left|U \chi_{E_{i}}\right|^{p} d \nu=\sum\left|c_{i}\right|^{r} \mu\left(E_{i}\right)=\|F\|_{r}^{r} .
\end{aligned}
$$

Thus $U$ is an isometry on all the spaces $L^{r}(X, \mu)$.

The last statement of the theorem follows from a result of Lamperti [4] which states that an operator which is an isometry on $L^{r}$ for two distinct values of $r$ must be of the form given above. This may also be deduced from the observation that the set map $\tau$ defined by $U \chi_{s}=\chi_{\tau(\Delta)}$ is Boolean.

Lamperti's theorem holds even if $\mu(X)=\mu(Y)=\infty$, while Theorem 3 does not. Theorem 3 may therefore be regarded as a partial generalization of Lamperti's result. Robert Strichartz has pointed out that the hypothesis $\mu(X)=\mu(Y)$ in Theorem 3 is essential. For, take $X=[0,1], Y=[0,2]$, and $\mu, \nu$ Lebesque measures. Let $(T f)(2)=(1 / 2) f((1 / 2) x)$. Then $T$ is an isometry on $L^{1}(X, \mu)$, but $\|T\|_{p}=2^{1-p / p}$.

Corollary. Let $\mu(X)=\nu(Y)<\infty$ and $1 \leqq p, q \leqq \infty, p \neq q$. Suppose $T$ is an isometry of $L^{p}(X, \mu)$ onto $L^{p}(Y, \nu)$ such that for all $F$ in $L^{q}(X, \mu),\|T F\|_{q} \leqq\|F\|_{q}$. Then $T$ is an isometry of each space $L^{r}(X, \mu)$ onto $L^{r}(Y, \nu), 1 \leqq r \leqq \infty$.

Proof. For $p>q$ this is Theorem 3. For $p>q$, apply Theorem 3 to $T^{*}$, which is an isometry on $L^{p^{\prime}}$ and a contraction on $L^{q^{\prime}}$, where $L^{p^{\prime}}$ and $L^{q^{\prime}}$ are the conjugate spaces of $L^{p}$ and $L^{q}$ respectively (so $\left.p^{\prime}<q^{\prime}\right)$.

THEOREM 4. If $G_{1}$ and $G_{2}$ are compact Abelian groups, and $L^{p}\left(G_{1}\right), L^{p}\left(G_{2}\right)$ are defined with respect to Haar measures, then Theorem 2 is valid when the hypothesis that $T$ be an isometry is replaced by the hypothesis that $\|T\| \leqq 1$.

Proof. We show that a convolution-preserving contraction of 
$L^{p}\left(G_{1}\right)$ onto $L^{p}\left(G_{2}\right), p \neq 2, \infty$, is automatically an isometry.

It is well known that any convolution-preserving operator must send characters onto characters. (For a quick proof, note that $\gamma$ is a character if and only if $\gamma * \gamma=\gamma$ and $\gamma * F$ is a scalar multiple of $\gamma$ for every $F \in L^{p}$.) Since the characters on a group form an orthonormal basis for $L^{2}$ of the group, $T$ is an isometry from $L^{2}\left(G_{1}\right)$ onto $L^{2}\left(G_{2}\right)$, and the corollary applies.

REMARKS 1. The analogues of Theorems 1 and 2 for $L^{2}$ are false. The falsity of Theorem 1 in this context is apparent from the example given in $\S 1$. And, Gaudry [3] has shown that there is a convolutionpreserving isometry from $L^{2}$ of the unit circle onto $L^{2}$ of the torus $\{(z, w)|| z|=| w \mid=1\}$, but these groups are certainly not topologically isomorphic.

2. Since this paper was submitted, [7] has appeared in which Theorems 1 and 2 are proved in slightly less generality.

3. The analogue of Theorem 2 for compact groups (with Haar measures) and $p=\infty$ may be found in [3] and [7].

I wish to thank Alessandro Figa-Talamanca for many interesting conversations and helpful suggestions. Thanks also go to G. Gaudry, $R$. Strichartz, and the referee for suggestions which materially improved the presentation.

\section{REFERENCES}

1. S. Banach, Theorie des Operations Lineaires, New York, 1955.

2. J. L. Doob, Stochastic Processes, New York, 1955.

3. G. I. Gaudry, Doctoral Dissertation, Canberra, Australla, 1955.

4. P. R. Halmos, Measure Theory, Princeton, N. J., 1950.

5. J. Lamperti, On the isometries of certain function spaces, Pacific J. Math. 8 (1958), 459-466.

6. C. E. Rickart, General Theory of Banach Algebras, Princeton, N. J., 1960.

7. R. S. Strichartz, Isomorphisms of convolution algebras, Proc. Amer. Math. Soc. 17, 858-862.

8. J. G. Wendel, On isometric isomorphisms of group algebras, Pacific J. Math. 1 (1951), 305-312.

9. - Left centralizers and isomorphisms of group algebras, Pacific J. Math. 2 (1952), 251-261.

Received August 8, 1966.

Massachusetts Institute of Technology 


\section{PACIFIC JOURNAL OF MATHEMATICS}

\section{EDITORS}

H. ROYDEN

Stanford University

Stanford, California

\section{J. P. Jans}

University of Washington

Seattle, Washington 98105

\section{J. DUGUNDJI}

Department of Mathematics University of Southern California Los Angeles, California 90007

\section{RICHARD ARENS}

University of California

Los Angeles, California 90024

\section{ASSOCIATE EDITORS}

E. F. BECKENBACH

B. H. NEUManN

F. WOLF

K. YosIDA

\section{SUPPORTING INSTITUTIONS}

UNIVERSITY OF BRITISH COLUMBIA CALIFORNIA INSTITUTE OF TECHNOLOGY UNIVERSITY OF CALIFORNIA MONTANA STATE UNIVERSITY UNIVERSITY OF NEVADA NEW MEXICO STATE UNIVERSITY OREGON STATE UNIVERSITY UNIVERSITY OF OREGON OSAKA UNIVERSITY UNIVERSITY OF SOUTHERN CALIFORNIA
STANFORD UNIVERSITY

UNIVERSITY OF TOKYO UNIVERSITY OF UTAH WASHINGTON STATE UNIVERSITY UNIVERSITY OF WASHINGTON AMERICAN MATHEMATICAL SOCIETY CHEVRON RESEARCH CORPORATION TRW SYSTEMS NAVAL WEAPONS CENTER 


\section{Pacific Journal of Mathematics}

\section{Vol. 25, No. $1 \quad$ September, 1968}

Glen Eugene Bredon, Cosheaves and homology................... 1

Robin Ward Chaney, A chain rule for the transformation of integrals in

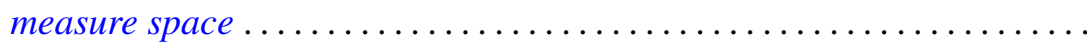

Colin W. Clark, On relatively bounded perturbations of ordinary differential operators................................... 59

John Edwin Diem, A radical for lattice-ordered rings.............. 71

Zeev Ditzian, On a class of convolution transforms ................ 83

Dennis Garoutte and Paul Adrian Nickel, A note on extremal properties characterizing weakly $\lambda$-valent principal functions............. 109

Shwu-Yeng Tzeng Lin, Fixed point properties and inverse limit spaces . . . 117

John S. Lowndes, Some dual series equations involving Laguerre polynomials ................................. 123

Kirti K. Oberai, Sum and product of commuting spectral operators ....... 129

J. N. Pandey and Armen H. Zemanian, Complex inversion for the generalized convolution transformation..................... 147

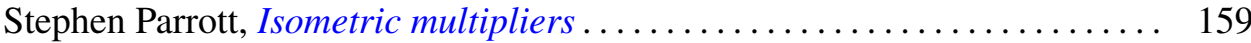

Manoranjan Prasad, Note on an extreme form .................. 167

Maciej Skwarczyński, A representation of a bounded function as infinite product in a domain with Bergman-Shilov boundary surface ......... 177

John C. Taylor, The Šilov boundary for a lattice-ordered semigroup ...... 185 Donald Reginald Traylor and James Newton Younglove, On normality and pointwise paracompactness ............................. 193

L. Tzafriri, Quasi-similarity for spectral operators on Banach spaces ... 